\title{
Adherence to Antiretroviral Therapy and Associated Factors Among Adult ARV Users in Arba Minch Hospital, Southern Ethiopia
}

\author{
Nuredin Nassir Azmach \\ Department of Statistics, College of Natural Sciences, Arba Minch University, Arba Minch, Ethiopia
}

Email address:

nurnas20@gmail.com

\section{To cite this article:}

Nuredin Nassir Azmach. Adherence to Antiretroviral Therapy and Associated Factors Among Adult ARV Users in Arba Minch Hospital, Southern Ethiopia. Central African Journal of Public Health. Vol. 3, No. 2, 2017, pp. 19-26. doi: 10.11648/j.cajph.20170302.12

Received: June 14, 2017; Accepted: June 28, 2017; Published: July 21, 2017

\begin{abstract}
The provision of Antiretroviral Therapy (ART), HIV/AIDS is becoming a chronic manageable disease; therefore to manage chronic disease, adherence to HIV medication is very important but a variety of other factors may complicate ART adherence that needs devotion from patients, provisions of health services and health care professionals, and having social support from the society. The purpose of this study was to determine the level of adherence to ART and associated factors among adult antiretroviral drugs (ARV) users. A cross sectional study was carried out at Arba Minch Hospital from March 5 to May 5, 2015. One month patients' self-report and pharmacy refill records were used to assess adherence. Data were collected by a standard questionnaire after pre-tested and data abstraction format. The collected data were entered in to Epi-Info and it was exported in to SPSS for data analysis. Multiple logistic regressions analysis was applied and statistical significance test was declared at P-value $<0.05$ and OR with $95 \%$ CI. Based on patients' self-report dose adherence, among the 428 study participants, the magnitude of adherence to ART in a month before interview was $77.10 \%$. Multivariable analysis showed that, adherence was positively associated with sex (male) (AOR=3.03, CI (1.69-5.42)), free from substance uses $(\mathrm{AOR}=3.49$, $\mathrm{CI}$ (1.80-6.77)), absence of side effect of drugs (AOR =2.61, CI (1.19-5.73)), ART schedule fit to daily routines (AOR=2.93, CI (1.24-6.91)) and feeling comfort on taking ART drug in front of others (AOR=3.32, CI (1.54-7.16)). The ART adherence rate of this study was relatively low compared with WHO standard and others study in Ethiopia. Sex, feeling comfort on taking the ART drugs, ART schedule fit to daily routines, substance use and drug side effects were strong predictors of adherence.
\end{abstract}

Keywords: ART, Adherence, HIV Patients, Southern Ethiopia

\section{Introduction}

Acquired Immune-Deficiency Syndrome (AIDS) is unique in human history in its rapid spread, extent and the depth of impact. The world has struggled to come to controls with its extraordinary dimension since the diagnosis of first AIDS case in 1981 [1].

Worldwide, more than 35 million people living with human immunodeficiency virus (HIV). In 2013 alone, an estimated of 2.1 million people were newly infected [2].

In Sub-Saharan countries more than two-third of (24.7 million) people living with HIV/AIDS (PLWHA). In 2013, an estimated 1.5 million people in the region became newly infected. Also an estimated 1.1 million people have died of AIDS, which accounted for $73 \%$ of the world's AIDS deaths [2].

In Ethiopia, the national adult HIV prevalence in 2013 was $1.3 \%$; and higher prevalence was reported among women $(1.8 \%)$ than men $(0.9 \%)$. In this year, 734,048 people were living with HIV among which 18,384 were new cases, and there were 34,365 HIV related mortalities occurred in the adult population [3].

Worldwide, to reduce deaths related with HIV/AIDS, we make use of Antiretroviral Therapy (ART), which was first introduced in the United States of America (USA) as a combination therapy in 1996; with the goals of achieving maximal and durable reduction in plasma viral load and restoration of immunological function [4]. Globally, around 12.9 million PLWHA (37\% of the total) had access to ART in 2013 [2].

In Ethiopia, the national Antiretroviral Drugs (ARV) policy was developed in 2002, and ART was first offered in July 2003 with the guideline for adults and adolescents; in the same year a fee-based ART program was officially started. However a fee-based ART program was changed into a free ART program in 2005 [5]. Consequently, the numbers of PLWHA accessing ART have dramatically increased from 8,000 in 2005 to over 350,000 in 2014 [6].

However, with the provision of ART, HIV/AIDS is 
becoming a chronic manageable disease [7]; thereby to manage chronic disease feature like adherence to HIV medication is an extremely important but a variety of other factors may complicate ART adherence that needs devotion from patients, provisions of health services and health care professionals, and having social support from the society [8].

Drug adherence is very important in progression of disease such as, to decrease the drug resistance, to improve the body's ability to fight HIV infection, to protect individuals from susceptibility to opportunistic infection, to decreases the viral load in the body and prolonged viral suppression [9]. Therefore, to obtaining the intended therapeutic effect, patients must have $\geq 95 \%$ adherence level is necessary [10].

Poles apart levels of adherence were reported in varies countries. The majority of the studies conducted in developed countries showed that the level of adherence $<75 \%$ (with the range of $20-100 \%$ ), whereas in developing countries report showed that the level of adherence was $>75 \%$ (with the range of $45-100 \%$ ) [11].

In Sub-Saharan countries ARV drug users, the prevailing level of adherence around $77 \%$ with the range of $68 \%$ up to $85 \%$ [12]. In Ethiopia, the level of adherence (62\%) reported in Mekelle [13], 72.4\% in Jimma [14], 77.9\% in Nekemt [15], $82.7 \%$ in Gondar [16], and $90 \%$ in Dessie [17]. All of the above level of adherence indicated that below the $95 \%$ (range $62-90 \%$ ); therefore, PLWHA requires long term treatment with adherence to medication for the success of the therapy.

Consistent factors for poor adherence include, patient related factors: socio-demographic and economic characteristics, patient skills, knowledge and psychosocial parameters; treatment regimen related factors: duration of treatment years, drug side effects and pill burden; environmental and social factors: supervision of treatment, social support and HIV related stigma; and the patient provider relationship related factors [7, 18].

Recently, HIV infected patients significantly do not reach high levels of adherence $(\geq 95 \%)$. So this can lead to devastating public health problems in resource limited countries [18]. Patients admitted at Arba Minch Hospital (AMH) that are on ART ward have history of sub-optimal adherence to treatment based on monthly follow up reports. As such this has to be looked into to find out what are factors associated with sub-optimal adherence.

The assessment of patients' adherence to treatment will enable to identify major problems to taking medication, for policy makers, researchers, and ultimately to prevent treatment failure. The study will also be supportive for program managers and decision makers working on HIV/AIDS area for proper designing and planning. Therefore, this study aimed to assess adherence to ART and associated factors among adult HIV patients in Arba Minch Hospital, Southern Ethiopia.

\section{Methods}

\subsection{Study Design and Setting}

A hospital based cross sectional study was conducted from March 5 to May 5, 2015 in Arba Minch Hospital, which is located in Arba Minch town, Gamo Gofa Zone. The town is located in Southern Nations Nationality and Peoples Region of Ethiopia (SNNPR) about $505 \mathrm{~km}$ South of Addis Ababa. The total population of the town was estimated to be 103,355 (in 2014). The hospital provides general health care service and higher levels of clinical care for the catchment populations and it has been providing HAART for thousands of HIV/AIDS adults and paediatric patients since 2004 come from rural and urban areas. Currently, there are 2556 ART users on follow up, among which 1511 adult patients.

\subsection{Source and Study Population}

The source populations were all adult HIV/AIDS patients who started HAART and have been on follow up in the hospital. Study populations were all HIV/AIDS adult outpatients systematically selected who receiving HAART and on follow up at the study setting whose age $\geq 18$ years. Patient who enrolled on ART less than 3 months period, pregnant, mental ill and missing clinical record were exclude from the study.

\subsection{Sample Size and Sampling Procedure}

The sample size was calculated using Epi-Info version 7 by using $90.83 \%$ proportion of adherence among nondepressed individuals with OR 2.5 [19]. The total sample size was 428. As to the sampling procedures, patients on follow up at ART treatment fulfilling the inclusion criteria were selected by using systematic random sampling method through appointments. There were 1511 adult HIV/AIDS patients on HAART treatment at AMH. The first participant was selected by using lottery method and then every third patients were taken among daily appointees depending on the order they come to the ART unit.

\subsection{Measurements}

The dependent variable in study was adherence to HAART. Adherence was measured by dose adherence: number of dose taken (number of doses prescribed minus number of dose missed) divided by number of doses prescribed multiplied by 100 [15]. When patients took $\geq 95 \%$ of their prescribed medication, we call it adherent; otherwise non-adherent [15]. In this study adherence was measured by two indicators of dose adherence i. e. based on one month patients' self-report of missing of the drug dose and regular following of monthly dispense schedule (pharmacy refill record).

The explanatory variables include socio-demographic factors: sex, age, residence, religion, educational level, employment status, monthly income, marital status and ethnic group; patients and social factors: social support, depression, substance use, disclosed HIV status, using reminder aids and ART knowledge (benefit and adherence); and treatment and clinical factors: duration of treatment, perceived medication side effect, CD4 count (initial and resent), other medications in addition to ARV, ART schedule fitness to daily routines, and feeling comfort on taking ART drug in front of others.

\subsection{Data Collection Procedure and Tools}

Data were collected using a pre-tested interviewer administered questionnaire technique and data abstraction 
format to obtain patient information chart from pharmacy refill data. The questionnaire was adapted from different literature that was used for data collection purpose. There were three data collectors and two supervisors who are health professionals experienced on ART services were participated. The investigator offered one day training for all data collectors and supervisors. Training was included explanation of the study objectives, techniques for interviewing, how to approach potential respondents and how to keep confidentiality later.

Patients interview which consists of socio-demographic characteristics, patients and social variables, treatment and clinical variables, the number of doses missed within a month, and reasons for missing the drugs. Patient information sheets involved to collect additional clinical information on CD4 cell count at enrolment and most recently, duration of treatment, co-administered drugs (other than ARV drugs), and monthly dispense schedule date were reviewed and recorded.

\subsection{Data Quality Control}

Pre-testing was done on 21 patients $(5 \%$ of the sample size) in Sikela Health Centre, Arba Minch, prior to the actual study in order to ensure all the data collectors understood each question in a similar way and collect the intended information. After the pre-testing some modification and improvement of the tool was done. The author and supervisors were performed immediate supervision on a daily basis. Each and every completed questionnaire was checked for completeness.

\subsection{Data Analysis}

Data were coded and entered in to Epi-Info version 3.3.2 and exported to SPSS-for windows version 20 for analysis. Descriptive statistics was done to describe the percentages and frequency distributions of the respondents by sociodemographic variables and others characteristics in the study.

Bivariate logistic regression analysis was done to determine the presence and degree of association between dependent and explanatory variables. Odds Ratios (OR) with 95\% Confidence Intervals (CI) and p-value less than 0.05 were determined to assess presence of statistically significant association between outcome and independent variables. Multicollinerity between independent variables was checked using residual error. Factors that show association in bivariate analysis and which has P-value less than 0.25 were included in the final model to control the effect of confounding and to identify significant factors.

In addition, Multivariate logistic regression model was constructed among the outcome and the explanatory variables to identify the significant predictors with Backward Stepwise selection method. The p-value of HosmerLemeshow test of Goodness-of-fit was 0.78 (p-value >0.05), so this indicate that the model was fit. At the end, adjusted OR with $95 \% \mathrm{CI}$, variables having p-value less than 0.05 were treated as showed a statistically significant association with adherence.

\subsection{Ethical Consideration}

The ethical approval and clearance was obtained from
Arab Minch University College of medicine and health science, ethical review committee. Before interviewing the patients, all the participants were given an explanation about the aim of the study and verbal informed consent was secured. In addition they were told that they had the right to terminate or refuse to participate in the study. In order to protect the confidentiality of the information obtained from respondents was kept confidential by using codes instead of any personal identifiers and privacy was maintained by independently answering the questionnaire.

\section{Results}

\subsection{Demographic Characteristics of Respondents}

Table 1. Socio-demographic characteristics PLWHA on HAART at Arba Minch Hospital, Gamo Gofa Zone, Southern Ethiopia, 2015.

\begin{tabular}{|c|c|c|c|}
\hline Variables & & Frequency & $\%$ \\
\hline \multicolumn{4}{|l|}{ Sex } \\
\hline & Male & 207 & 48.4 \\
\hline & Female & 221 & 51.6 \\
\hline \multicolumn{4}{|l|}{ Age } \\
\hline & $20-30$ & 98 & 22.9 \\
\hline & $31-39$ & 166 & 38.8 \\
\hline & $40-49$ & 133 & 31.1 \\
\hline & $\geq 50$ & 31 & 7.2 \\
\hline \multicolumn{4}{|l|}{ Residence } \\
\hline & Urban & 341 & 79.7 \\
\hline & Rural & 87 & 20.3 \\
\hline \multicolumn{4}{|l|}{ Ethnicity } \\
\hline & Gamo & 246 & 57.5 \\
\hline & Gofa & 41 & 9.6 \\
\hline & Wolayta & 61 & 14.3 \\
\hline & Amhara & 37 & 8.6 \\
\hline & Gurage & 15 & 3.5 \\
\hline & Konso & 9 & 2.1 \\
\hline & Others* & 17 & 4.4 \\
\hline \multicolumn{4}{|l|}{ Religion } \\
\hline & Orthodox & 251 & 58.6 \\
\hline & Protestant & 170 & 39.7 \\
\hline & Muslim & 7 & 1.6 \\
\hline \multicolumn{4}{|c|}{ Marital status } \\
\hline & Single & 63 & 14.7 \\
\hline & Married & 197 & 46.0 \\
\hline & Windowed & 63 & 14.7 \\
\hline & Divorced/Separated & 105 & 24.6 \\
\hline \multicolumn{4}{|c|}{ Education status } \\
\hline & Not read and write & 67 & 15.7 \\
\hline & Read and write & 47 & 11.0 \\
\hline & Primary & 157 & 36.7 \\
\hline & Secondary & 75 & 17.5 \\
\hline & Diploma and above & 82 & 19.1 \\
\hline \multicolumn{4}{|c|}{ Occupation } \\
\hline & $\begin{array}{l}\text { Employed by } \\
\text { family member }\end{array}$ & 182 & 42.5 \\
\hline & $\begin{array}{l}\text { Employed by } \\
\text { nonfamily member }\end{array}$ & 53 & 12.4 \\
\hline & Self employed & 193 & 45.1 \\
\hline \multicolumn{4}{|l|}{ Income** } \\
\hline & $<1000$ & 205 & 56 \\
\hline & $1000-3000$ & 128 & 34 \\
\hline & $>3000$ & 37 & 10 \\
\hline
\end{tabular}

*Tigree, Oromo, Zeysse, and Hadya; ** Number of participants having income $(n=370)$ 
A total of 428 adult PLWHA participated in this study giving a response rate of $100 \%$, of these, $166(38.8 \%)$ were in the age group 31-39 years and the mean (SD) age of the respondents were $36.98( \pm 8.32)$ ranging from 20 to 66 years and females constitute $221(51.6 \%)$. Among the study participants $341(79.7 \%)$ were living in urban area and the rest were rural area. Two hundred fifty one $(58.6 \%)$ of the clients were orthodox by religion followed by protestant (39.7\%). The ethnicity of the respondents $246(57.6 \%)$ were Gamo. One hundred ninety seven $(46 \%)$ of the participants were married; among this, all were living with their spouse, the remaining $54 \%$ living with their family, friends or alone. One hundred fifty seven $(36.7 \%)$ were attended primary school. One hundred ninety three $(45.1 \%)$ were merchant/business worker. The majority of participants, $370(86.4 \%)$ had monthly income. The minimum monthly income was 70.00 birr while maximum monthly income was 5,337.00 birr (Table 1).

\subsection{Patients and Social Related Variables}

Four hundred sixteen (97.2\%) had disclosed their HIV status to their family members, friends or work mate. The majority of the participants $344(80.4 \%)$ were getting social support. Out of this participants were helped by their family members, friends or work mate on remembering to take their medications, material and financial support, and advising, which were 276 (64.49\%), 186 (43.46\%), and 71 (16.58\%), respectively. Three hundred thirty four $(78 \%)$ of subjects were free of any substance abuse but 57 (13.32\%) drink alcohol, $23(5.37 \%)$ use kcat, $2(0.47 \%)$ smoking cigarette and $12(2.84 \%)$ use the above substances in some combination. Seventy five $(17.5 \%)$ of clients were found to be depressed. The preponderance of them had no depression status $353(82.5)$.

\subsection{Patients' Adherence Support strategies}

Almost the entire participants 421 (98.4\%) were used at least one strategy to remind themselves to take their medication on time, among this, $85.27 \%$ set the mobile phone alarm (Table 2).

Table 2. Adherence support strategies PLWHA on HAART at Arba Minch Hospital, Gamo Gofa Zone, Southern Ethiopia, 2015.

\begin{tabular}{lll}
\hline Variable & Frequency & \% \\
\hline Strategies for supporting adherence (421)* & & \\
\hline Set the mobile phone alarm & 359 & 85.27 \\
Adjust other persons to remind them & 261 & 61.99 \\
Placed pills in an open space & 61 & 14.49 \\
Carried pills in pocket/handbag when leave home & 23 & 5.46 \\
\hline
\end{tabular}

* More than one answer is possible

\subsection{Treatment and Clinical Related Characteristics}

As to the treatment durations, $243(56.8 \%)$ of the participants were more than 5years on ART, with a mean (SD) of 5.6 (2.5) years (range of 4 month to 12 years). The CD4 counts before starting ART, 220 (51.4\%) of participants had CD4count $<200$ cells $/ \mathrm{mm} 3$ where as the most recent CD4 counts, among the participants, only $9(2.1 \%)$ of them had CD4 count $<200$ cells/mm3. About forty four $(10.3 \%)$ patients responded that they have side effects of ARV drugs in the previous one month prior to data collection time. The commonest reported side effects were nausea and vomiting $19(30.65 \%)$, pain and numbness $13(20.96 \%)$, skin rash 12 $(19.35 \%)$, headache $9(14.52 \%)$, fever or chills $6(9.68 \%)$ and others $3(4.84 \%)$. Two hundred eighty seven $(80.4 \%)$ of the participants not taken other mediation in addition to ARV drugs (Table 3).

Table 3. Treatment and clinical related variables PLWHA on HAART at Arba Minch Hospital, Gamo Gofa Zone, Southern Ethiopia, 2015.

\begin{tabular}{|c|c|c|c|}
\hline Variables & & Frequency & $\%$ \\
\hline \multicolumn{4}{|c|}{ Duration of treatment in year } \\
\hline & $\leq 5$ & 185 & 43.2 \\
\hline & $>5$ & 243 & 56.8 \\
\hline \multicolumn{4}{|c|}{ CD4 count before treatment } \\
\hline & $\leq 200$ & 220 & 51.4 \\
\hline & $>200$ & 208 & 48.6 \\
\hline \multicolumn{4}{|c|}{ Recent CD4 count } \\
\hline & $\leq 200$ & 9 & 2.1 \\
\hline & $201-499$ & 113 & 26.4 \\
\hline & $\geq 500$ & 306 & 71.5 \\
\hline \multicolumn{4}{|c|}{ Side effect of drugs } \\
\hline & Yes & 44 & 10.3 \\
\hline & No & 384 & 89.7 \\
\hline \multicolumn{4}{|c|}{$\begin{array}{l}\text { Other medications in addition } \\
\text { to ARV }\end{array}$} \\
\hline & Yes & 41 & 9.6 \\
\hline & No & 387 & 90.4 \\
\hline \multicolumn{4}{|c|}{$\begin{array}{l}\text { ART schedule fitness to daily } \\
\text { routines }\end{array}$} \\
\hline & Yes & 366 & 85.5 \\
\hline & No & 62 & 14.5 \\
\hline \multicolumn{4}{|c|}{$\begin{array}{l}\text { Feeling comfort on taking ART } \\
\text { drug in front of others }\end{array}$} \\
\hline & Yes & 334 & 80.4 \\
\hline & No & 84 & 19.4 \\
\hline \multicolumn{4}{|c|}{ ART benefit knowledge } \\
\hline & Good & 347 & 81.07 \\
\hline & Unsatisfactory & 81 & 18.93 \\
\hline \multicolumn{4}{|c|}{ ART adherence knowledge } \\
\hline & Good & 361 & 84.34 \\
\hline & Unsatisfactory & 67 & 15.66 \\
\hline
\end{tabular}

\subsection{Adherence to Self-Report vs Adherence to Monthly Dispense Schedule}

In the study, based on patients' self-report dose adherence, $330(77.10 \%)$ of the study subjects were adhered strictly to their medication while $369(86.21 \%)$ of the study subjects were adherent to regular following of monthly dispense schedule (pharmacy refill record). 


\subsection{Reasons for Missing Doses}

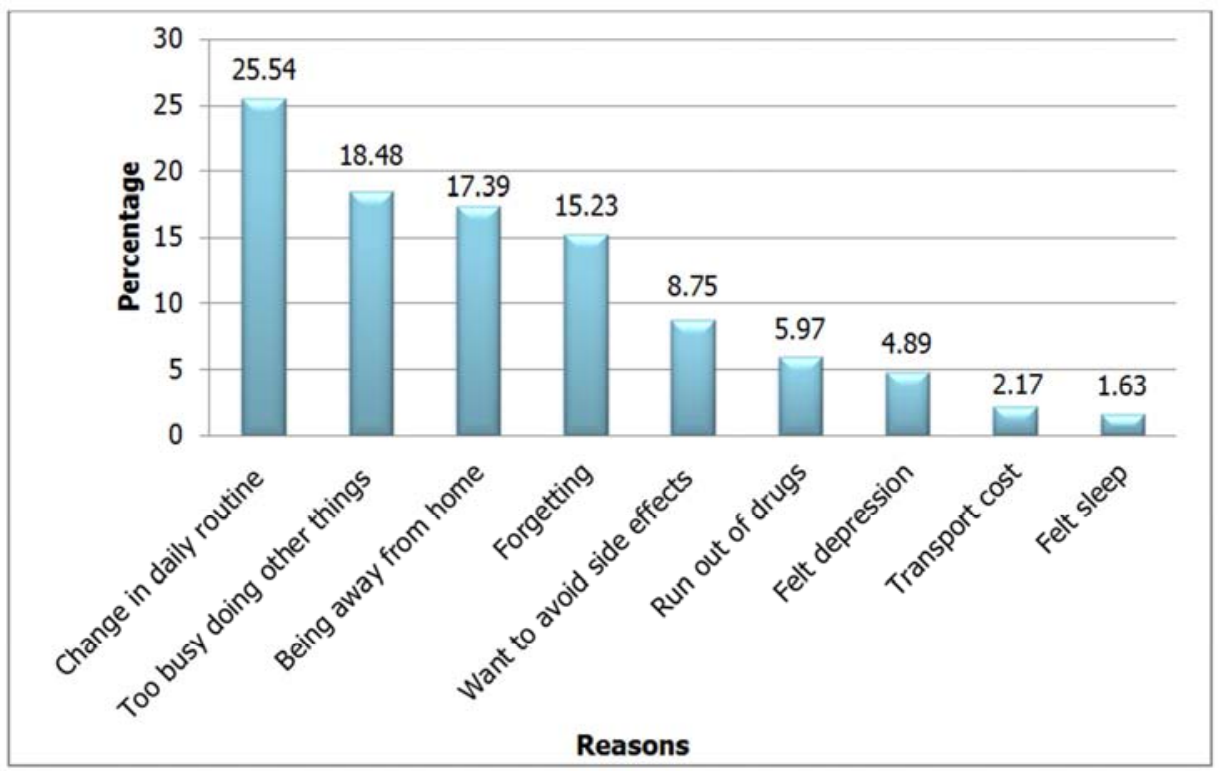

Figure 1. Reasons for missing ART medications by PLWHA at Arba Minch Hospital, Gamo Gofa Zone, Southern Ethiopia, 2015.

The principal reasons reported for skipping doses in this study were change in their daily routine work 47 (25.54\%), busy with doing other things $34(18.48 \%)$ and being away from home $32(17.39 \%)$, and the rest reasons included simply forgot to take the pills, want to avoid side effects, run out of drugs, felt depression, transport cost, and felt sleep (Figure 1).

\subsection{Factors Associated with Adherence to ART}

Table 4. Multivariable analysis between explanatory and adherence to HAART among study participants at Arba Minch Hospital, Gamo Gofa Zone, Southern Ethiopia, 2015.

\begin{tabular}{|c|c|c|c|c|}
\hline \multicolumn{5}{|c|}{ Adherence statues } \\
\hline \multirow[b]{2}{*}{ Explanatory Variables } & \multirow{2}{*}{$\begin{array}{l}\text { Adherent } \\
\text { (1) }\end{array}$} & \multirow{2}{*}{$\begin{array}{l}\text { Non Adherence } \\
\text { (0) }\end{array}$} & \multicolumn{2}{|c|}{ OR $(95 \%$ CI $)$} \\
\hline & & & Crude & Adjusted \\
\hline \multicolumn{5}{|l|}{ Sex } \\
\hline Male & $170(51.5)$ & $37(37.8)$ & $1.75(1.11-2.78)$ & $3.03(1.69-5.42)$ \\
\hline Female & $160(48.5)$ & $61(62.2)$ & 1 & 1 \\
\hline \multicolumn{5}{|l|}{ Active substance Use } \\
\hline No & $265(80.3)$ & $69(70.4)$ & $1.71(1.03-2.86)$ & $3.49(1.80-6.77)$ \\
\hline Yes & $65(19.7)$ & $29(29.6)$ & 1 & 1 \\
\hline \multicolumn{5}{|l|}{ Side effect of drugs } \\
\hline No & $306(92.7)$ & $78(79.6)$ & $3.27(1.72-6.22)$ & $2.61(1.19-5.73)$ \\
\hline Yes & $24(7.3)$ & $20(20.4)$ & 1 & 1 \\
\hline \multicolumn{5}{|c|}{ ART schedule fitness to daily routines } \\
\hline Yes & $304(92.1)$ & $62(63.3)$ & $6.79(3.83-12.05)$ & $2.93(1.24-6.91)$ \\
\hline No & $26(7.9)$ & $36(36.7)$ & 1 & 1 \\
\hline \multicolumn{5}{|c|}{ Feeling comfort on taking ARV in front of others } \\
\hline Yes & $289(87.6)$ & $55(56.1)$ & $5.51(3.29-9.23)$ & $3.32(1.54-7.16)$ \\
\hline No & $41(12.4)$ & $43(43.9)$ & 1 & 1 \\
\hline
\end{tabular}

By applying bivariate logistic regression analyses, the results indicated that from socio-demographic variables, age ( $\geq 50$ year) and sex(male) of the participant were significantly associated with adherence $(\mathrm{OR}=3.92 ; 95 \% \mathrm{CI}$ : 1.11,13.93) and $(\mathrm{OR}=1.75 ; 95 \% \mathrm{CI}: 1.11,2.78)$, respectively. The characteristics of personal behaviour, treatment and clinical related variables of the study participants were associated with ART adherence status. Active substance use $(\mathrm{OR}=1.71$; 95\%CI: 1.03,2.86), depression status (OR=0.31; 95\%CI: $0.18,0.53)$, drug side effect $(\mathrm{OR}=3.27 ; 95 \% \mathrm{CI}: 1.72,6.22)$, resent CD4 count $(201-499$ cells $/ \mathrm{mm} 3)(\mathrm{OR}=0.22 ; 95 \% \mathrm{CI}$ : $0.06,0.84)$, ART schedule fitness to daily routines $(\mathrm{OR}=6.79$; 95\%CI: 3.83,12.05) and feeling comfort on taking ART drug in front of others $(\mathrm{OR}=5.51$; 95\%CI: 3.29,9.23). Therefore, the variables like age, sex, active substance uses, side effect, depression, resent CD4 count, ART schedule fitness to daily routines, and feeling comfort on taking ART drug in front of others were included in the final model. In addition, place of residence and social support were included in the final model because of $p$-value $<0.25$ which was 0.15 and 0.16 , respectively. Multivariate logistic regression showed that patients who do not use active substance were $(\mathrm{AOR}=3.49$; 95\% CI: $1.80,6.77$ ), and other variables included sex, drug side effect, feeling comfort on taking ART drug in front of others, and ART schedule fitness to daily routines were statistically significantly associated with ART adherence (Table 4). 


\section{Discussion}

In the study, participants' self-reports of dose adherence indicated sub-optimal dose adherence $(77.1 \%)$ in last month prior to the study period, while higher adherence to monthly dispense adherence (86.21\%) in comparison with the patients' self-report adherence. Participants were more adherences to monthly dispensing schedule this might be because they have remainder cards for monthly appointments.

As it is mentioned to the above, the patients' dose adherence in the study area was (77.1\%) which is almost consistent with the study done in Nekemte Hospital (77.9\%) [15]. However, lower than that reported in Dessie referral Hospital (90\%) [17]. This may suggest that patients having low socioeconomic status were able to achieve good rates of adherence with ART. Almost a total of $(97 \%)$ the Dessie referral Hospital study participant reported income of less than 1000 birr per month [17], whereas $54 \%$ of this study participant earned less than 1000 birr per month. The finding of this study, monthly dispense schedule assessment of adherence of $86.21 \%$ was below $95 \%$ of adherence expected of these participants but it is greater than the finding of Nigeria [20] in Sobi Specialist Hospital (70.9\%).

In this research, sex, presence of side effect in the past four weeks, active substance use, fitness of daily treatment schedule with daily routine, and feeling comfort on taking ART drug in front of others were all significantly associated with adherence.

Among socio demographic variables, sex was found to be significantly associated with adherence. Male participants were about 3 times more likely to be adherent than the female respondents. From the researcher view of point, what it might cause the female participants were busy to work with burden of routine daily household activities which might have contributed to forgetting and as a consequence of missing daily dose. These female patients also experience gender related barriers to accessing HIV care services, thus affecting ART adherence. For instance, many females have to obtain permission from a male or a relative to seek health care, which is problematic when females have to ask for money and take time away from household tasks.

But, in this finding, the study is in contradiction with the finding that was conducted in Zambia [21], where female participants had good responses to treatment and may have had greater motivation to adhere to ART compared with that of male participants (AOR $=3.3 ; 95 \% \mathrm{CI}, 1.2-8.9$ ), as suggested that female participants caring for children emphasize their role as primary care to children and their children are known to be a facilitating factor in adherence among them.

In the study, there was statistically a significant association between substance use and ART adherence status. Subjects with no substance use have more than three times more adherent than those with substance users, and more frequently observed in male respondents than in female respondents. In other studies, in Southern Ethiopia [22], Eastern Tanzania [23], India [24], and Nepal [25] not any substance uses has been an important predictor of adherence respectively. Patients' behaviours related to active substance uses and illegal drugs themselves may lead to poorer adherences to ART. Patients used the ARV drugs and active substances alternatively which directly leads to poor adherence and can result into treatment failure and development of drug resistance [24]. Active substance users would change their life style and sleeping pattern; therefore it can result in to forgetfulness of the dosing timing especially when they drunk excessive alcohol. In addition, too much alcohol use can cause liver damage, increase liver enzymes which lead to short drug's half-life than expected. Moreover, alcohol consumption was exacerbates ARV side effects [23].

Medication side effects of ARV effect in the past four weeks had statistically significance association with adherence. Participants with no side effect have more than two times more adherent than those with side effect. The most frequently reported side effects were nausea, vomiting and numbness on poor adherent participants. This finding was supported by a study conducted on HIV-patients in Nepal (95\% CI, 1.09-21.8), and Nigeria [20, 25] who used to skip treatment doses due to drug side effects. Medication side effects can therefore play a large role in disheartening adherence to therapy which in turn impacts the development of drug resistance and treatment failure [9]. Patients with a higher number of side effects were usually stop taking their drugs correctly because they are discouraged by side effects, so preparing patients for understanding of side effects is critical [7].

Furthermore, the management of side effects is a main issue of enhances the adherence status of ART patients. Hence, it might be necessary to educating patients because they may not be aware about side effects and health care providers should advice on managing about possible side effects at the commencement of treatment, although after start the treatment with patients. Therefore, patients are known to approach their health care providers when they start feeling that the medications are giving trouble this might be helping to identify the responsible ARV drug.

According to this finding, there was significant association that patients who reported to have fitness of treatment schedule with daily routine work were about three times more likely to be adherent than those who did not fit the treatment schedule and daily routine work. This is consistent with the findings of study conducted in Desee [17]. In addition, study in Vietnam showed that stable employment was associated with optimal adherence [26]. Patients whose daily treatment schedule fit with daily routine work were important to ART regimen adherence. For the reason that to prevent daily dose interruption in different reasons: daily treatment schedule and daily treatment have to concise each other. Therefore, health care providers need to work with the patient to draw up the plan that considers the everyday activities.

Patients who reported to have feeling comfort on taking ART drug in front of others were more than three times more likely to be adherent than those who feeling discomfort on taking ART drug in front of others, which agrees with the finding from the study done in Northwest Ethiopia (95\% CI, 4.6-28), where patients who reported having comfort on taking ART drugs in front of others have been shown to be a significant predictor of adherence [27]. This might be due to 
disclosure of the participants HIV status to their immediate member of family and society, hence this will enhance theme to gain confidence on themselves and the environment. In addition to this, patients have disclosures may enhance psychologically free from the facts that they gave no place from stigma and discrimination associated influences by masking the effect of stress and anxiety. However, patients fear of stigmatization by the community contributes to poor drug adherence, which some patients felt uncomfortable while taking ART drugs from the pharmacy, despite the place of pharmacy were similar to other patients in the hospital.

The most principal reasons of the participants cited were change in their daily routine work, being busy, being away from home, and forgetting in more than $75 \%$ of the cases. Similar reasons were reported in studies conducted in Nekemte hospital and Debre Markos referral hospital [15, 27]. These principal reasons assessed for an individual patient and appropriate adherence enhancing intervention may be undertaken to achieve the success of therapy. In this respect, health professionals might need to organize the plan that considers the everyday activities of patients, and adherence counseling might incorporate strategies on using of multiple memory aids to avoid simply forgetting to take pills.

On the other hand, other socio demographic variables like age and residence were not significantly associated with adherence. These result were agreed with others studies in Tanzania and Wolaita Sodo $[19,23]$ whereas these finding is contradicted with other study in India [24]. Also variables like social support, depression, and resent CD4 count were not significantly associated with adherence.

\section{Conclusions}

In general, the ART patients' adherence in this study was relatively low compared with WHO standard and other study in Ethiopia. According to this study, sex (male), feeling comfort on taking the ART drugs, ART schedule fitness to daily routines, substance use and drug side effect in the past four weeks prior to the study period were major contributing factors for ART adherence. The main reasons for missing dose(s) were change in their daily routine work, being busy, being away from home and forgetting. Based on the findings of the study the following recommendations were forwarded.

\subsection{For Health Professional}

To increase about ART adherence of the client, health provider should give education about adherence especially female patients; and on patients' behavioral change about active substance uses.

Health professional and adherence supporters should give advice at the commencement of ART service to increase patients' awareness of the side effects of ARV and manage them.

To fit the daily treatment schedule with the daily routine work, health care providers should work with the patients to draw up the plan that considers the everyday activities.

Adherence counseling and health information dissemination need to decrease missing doses include strategies to minimize forgetfulness by using multiple memory aids such as watch or mobile bell, placed pills in an open space, reminded by family members, friends or work mate, and carried pills in pocket or handbag when they leave home.

\subsection{For ART Users}

To maintain the success of therapy, ART users should be following the treatment instructions given by the health care providers.

\subsection{For Researchers}

Further study on adherence rate and its associated factors with multiple adherence measurements to resolve the barriers to adherence and it should be carried out in longitudinal base by integrating with qualitative study design to identify behavioral related factors.

\subsection{Limitation}

One of measurements of adherence was self-report, so there might be recall bias and social desirability bias when patients may be wont to over or under estimate their adherence status. Thus, the result of the study should be used carefully. Given this limitation our finding is significant to identify the factors associated with adherence and to improve the health of patients.

\section{Competing Interests}

The authors declare that they have no competing interest.

\section{Acknowledgements}

The author would like to acknowledge Arba Minch University, Institutional Review Board, Nech Sar Campus for giving the ethical clearance. We are greatly indebted to thank all respondents for their willingness to participate in the study. Also, our deepest gratitude goes to our data collectors and Arba Minch Hospital manager. The researchers also thank all individuals who have in one way or another contributed to the completion of this research.

\section{References}

[1] UNAIDS. Joint United Nations Program on HIV/AIDS Fact Gap Report. Geneva: UNAIDS, 2014.

[2] World Health Organization. Global estimation of HIV/AIDS. Geneva: WHO, 2014.

[3] World Health Organization: HIV/AIDS Estimates and Projections in Ethiopia, 2011-2016. Geneva: 2011.

[4] Palella J, Delaney K, Moorman A. Declining morbidity and mortality among patients with advanced human immunodeficiency virus infection. New Engl J Med. 1998; 338:853-60.

[5] Ethiopian Ministry of Health: Strategic plan for multi sectoral response against HIV/AIDS for 2004-2008. Addis Ababa (Ethiopia): Ministry of Health, 2004. 
[6] Ethiopian Ministry of Health: Access of Antiretroviral drugs and treatment program in Ethiopia. Addis Ababa (Ethiopia): Ministry of Health, 2014.

[7] Seema S, K. Srikanth R, Sampada D. Optimizing adherence to antiretroviral therapy. Indian J Med Res. 2011; 134:835-49.

[8] Sharada PW, Edwin VT, Padam S, Julian R, Susan B, Pamela $\mathrm{K}$, et al. Factors influencing adherence to antiretroviral treatment in Asian developing countries: a systematic review. Tropical Medicine and International Health. 2012; 17(1):7181.

[9] Bangsberg D. Less than 95\% adherence to non-nucleoside reverse transcriptase inhibitor therapy can lead to viral suppression. Clin Infec Dis. 2006; 43(7):939-41.

[10] Paterson DL, Swindells S, Mohr J, Brester M, Vergis E, Squire C, et al: Adherence to protease inhibitor therapy and outcomes in patients with HIV infection. Ann Intern Med 2000, 133:21-30.

[11] Braitstein P, Brinkhof M, Dabis F, Schechter M, Boull A, et al. Mortality of HIV-1-infected patients in the first year of antiretroviral therapy: Comparison between low-income and high-income countries. Lancet. 2006; 367:817-24.

[12] Chabikuli NO, Datonye DO, Nachega J, Ansong D. Adherence to antiretroviral therapy, virologic failure and workload at the Rustemburg Provincial hospital. SA Fam. Pract. 2010; 52(4): 350-355.

[13] Kiday H, Belachew E, Mussie A, Girmatsion F. Factors associated with adherence of highly active antiretroviral therapy among adult HIV/AIDS patients in Mekelle Hospital Northern Ethiopia. Science Journal of Public Health. 2014; 2(4):367-72.

[14] Ayele T, Tefera B, Fisehaye A, Sibhatu B. Predictors of adherence to antiretroviral therapy among people living with HIV/AIDS in resource-limited setting of southwest ethiopia. AIDS Research and Therapy. 2010; 7:39.

[15] Solomon HE, Wote AR, Mulugeta TA. Medication adherence and associated factors among patients on highly active antiretroviral therapy in Nekemte Hospital, Ethiopia. Gaziantep Med J. 2014; 20(3):199-208.

[16] Belay T, Fantahun B, Andargachew M, Assefa G, Frank E, Ulrich S. Magnitude and determinants of nonadherence and nonreadiness to highly active antiretroviral therapy among people living with HIV/AIDS in Northwest Ethiopia: a crosssectional study. AIDS Research and Therapy. 2010; 7(2):1-8.

[17] Birhanu D, Tesfahun C. Adherence to antiretroviral therapy and associated factors among patients living with HIV/AIDS in Dessie Referral Hospital, Northern Ethiopia. International Journal of Pharma Sciences and Research. 2014; 5(9):572-81.

[18] Ayalu AR, Sibhatu B. Determinants of Adherence to Antiretroviral Therapy among HIV-Infected Patients in Africa. Hindawi Publishing Corporation AIDS Research and Treatment. 2012; 1:1-8.

[19] Amsalu A, Wanzahun G, Mohammed T, Tariku D. Factors Associated with Antiretroviral Treatment Adherence among Adult Patients in Wolaita Soddo Hospital. Journal of Tropical Diseases. 2013; 1(4):7.

[20] Bello SI. HIV/AIDS Patients' Adherence to Antiretroviral Therapy in Sobi Specialist Hospital, Ilorin, Nigeria. Journal of Advanced Scientific Research. 2011; 2(3):52-57.

[21] Yuri S, Kazuhiro K, Christopher D, Izukanji S, Crispin M, Gardner S, et al. Adherence to antiretroviral therapy (ART) during the early months of treatment in rural Zambia: influence of demographic characteristics and social surroundings of patients. Annals of Clinical Microbiology and Antimicrobials. 2012; 11(34):11.

[22] Belay DB, Yemane B, Eskezyiaw AG, Direslgne MA. Determinants of None-adherence to antiretroviral therapy among HIV-infected adults in Arba Minch General Hospital, Gamo Gofa Zone, Southern Ethiopia: A case control study. American Journal of Health Research. 2014; 2(5):234-40.

[23] Boniphace I, Boniphace J, Ferdinand M, Tanner M. A casecontrol study of factors associated with non-adherent to antiretroviral therapy among HIV infected people in Pwani Region, eastern Tanzania. Tanzania Journal of Health Research. 2012; 14(3):1-12.

[24] Anant G, Meenakshi GN, Geeta S, Mohan KD. Adherence to Antiretroviral Therapy. Journal of API. 2012; 60:16-21.

[25] Shigdel R, Klouman E, Bhandari A, Ahmed LA. Factors associated with adherence to antiretroviral therapy in HIVinfected patients in Kathmandu District, Nepal. HIV/AIDS Research and Palliative Care. 2014; 6:109-16.

[26] Bach X, Long $T$, Nga $H$, Quynh VH, Jongnam $H$. Determinants of antiretroviral treatment adherence among HIV/AIDS patients: a multisite study. Glob Health Action. $2013 ; 6: 7$.

[27] Mulugeta A, Mekonnen A, Mulat A, Dube J. Level of ART Adherence and Associated Factors among HIV Sero- Positive Adult on Highly Active Antiretroviral Therapy in Debre Markos Referral Hospital, Northwest Ethiopia. Journal Antivirals Antiretrovirals. 2014; 6(3):120-26. 